\title{
Review of: "Structure and conservation of amyloid spines from the Candida albicans Als5 adhesin including similarity to human LARKS"
}

\author{
Abhigyan Nath
}

Potential competing interests: The author(s) declared that no potential competing interests exist.

The authors have vetted the structure and conservation of amyloid spines effectively.

The manuscript can be accepted. 